\title{
A forming, dust-enshrouded disk at $z=0.43$ : the first example of a massive, late-type spiral rebuilt after a major merger?
}

\author{
F. Hammer ${ }^{1}$, H. Flores ${ }^{1}$, Y. B. Yang ${ }^{1}$, E. Athanassoula ${ }^{2}$, M. Puech $^{3,1}$, M. Rodrigues ${ }^{1}$, and S. Peirani ${ }^{4}$ \\ 1 Laboratoire Galaxies Étoiles Physique et Instrumentation, Observatoire de Paris, 5 place Jules Janssen, 92195 Meudon, France \\ e-mail: francois. hammer@obspm.fr \\ 2 Laboratoire d'Astrophysique de Marseille, Observatoire Astronomique de Marseille Provence, Technopole de l'Étoile, \\ Site de Chateau-Gombert, 38 rue Frédéric Joliot-Curie, 13388 Marseille Cedex 13, France \\ 3 ESO, Karl-Schwarzschild-Strasse 2, 85748 Garching bei Munchen, Germany \\ ${ }^{4}$ Institut d'Astrophysique de Paris, France
}

Received 1 July 2008 / Accepted 27 November 2008

ABSTRACT

\begin{abstract}
By combining Ultra Deep Field imagery from HST/ACS with kinematics from VLT/GIRAFFE, we derive a physical model of distant galaxies in a way similar to that achievable for nearby galaxies. A significant part of the evolution in the density of cosmic star formation is related to the rapid evolution of both luminous IR galaxies (LIRGs) and Luminous Compact galaxies: here we study the properties of a distant, compact galaxy, J033245.11-274724.0, which is also a LIRG. Given the photometric and spectrophotometric accuracies of data of all wavelengths, we can decompose the galaxy into sub-components and correct them for reddening. Combination of deep imagery and kinematics provides a reasonable physical model of the galaxy. The galaxy is dominated by a dust-enshrouded disk revealed by UDF imagery. The disk radius is half that of the Milky Way and the galaxy forms stars at a rate of $20 M_{\odot} / y r$. Morphology and kinematics show that both gas and stars spiral inwards rapidly to feed the disk and the central regions. A combined system of a bar and two nonrotating spiral arms regulates the material accretion, induces high velocity dispersions, with $\sigma$ larger than $100 \mathrm{~km} \mathrm{~s}^{-1}$ and redistributes the angular momentum. The detailed physical properties of J033245.11-274724.0 resemble the expectations of modeling the merger of two equal-mass, gaseous-rich galaxies, $0.5 \mathrm{Gyr}$ after the merger. They cannot be reproduced by any combination of intrinsic disk perturbations alone, given the absence of any significant outflow mechanisms. In its later evolution, J033245.11-274724.0 could become a massive, late-type spiral that evolves to become part of the Tully-Fisher relation, with an angular momentum induced mostly by the orbital angular-momentum of the merger.
\end{abstract}

Key words. galaxies: formation - galaxies: spiral - Galaxy: kinematics and dynamics

\section{Introduction}

Disk galaxies constitute the majority of the galaxy population observed in the local universe. They represent $70 \%$ of intermediate-mass galaxies (stellar masses ranging from $3 \times$ $10^{10}$ to $3 \times 10^{11} M_{\odot}$ ), which themselves include at least twothirds of the present-day stellar mass density (e.g., Hammer et al. 2005). A model of disk formation developed by Fall \& Efstathiou (1980), assumed that formation results from gas cooling and condensation in dark haloes. Their angular momentum would have been acquired during early interactions (Mo et al. 1998) and later the disks may have grown smoothly by gas accretion. Such a model successfully reproduces flat rotation curves but faces a difficulty in reproducing the angular momentum of galactic disks: simulated galaxies have too small angular momentum (e.g., Steinmetz \& Navarro 1999). However, the secular model of disk formation is consistent with observational studies of the Milky Way. It originates from early studies of the Milky Way that developed into a general description of the formation of a disk galaxy embedded in a halo (e.g., Eggen et al. 1962). On the other hand, the peculiar nature of the Galaxy has been questioned (Hammer et al. 2007): compared to other spirals of similar rotational velocity, it exhibits too small stellar mass, angular momentum, disk radius and halo stars $[\mathrm{Fe} / \mathrm{H}]$ ratio. The Milky Way is possibly peculiar because it has not experienced any significant collisions since $10-11 \mathrm{Gyr}$, in contrast to M 31
(Ibata et al. 2005; Brown et al. 2008), whose properties are far more typical (Hammer et al. 2007). Thus, it could be useful to re-evaluate the secular model of disk formation.

An important debate is how to reconcile the presence of numerous present-day thin disks with their fragility to collisions (Toth \& Ostriker 1992). All recent studies now appear to agree that a significant fraction ( $40-90 \%)$ of local intermediate-mass galaxies have experienced a major merger within the past $8 \mathrm{Gyr}$ (Rawat et al. 2008; Ryan et al. 2008; Lotz et al. 2008a; Bell et al. 2006). Studies of the merger-rate evolution have now reached sufficient maturity: the pair fraction of galaxies at $z \sim 0.6$ with a mass ratio ranging from 1:1 to $1: 4$ is $5 \pm 1 \%$, regardless of the adopted observational methodology (e.g. Bell et al. 2006, and references therein). The main uncertainty presently exists in the modelling, i.e. the assumed characteristic time for a true pair to actually merge, but there are few doubts that major mergers play an important role in shaping present-day galaxies and thus, the Hubble sequence.

The above considerations may support the Hammer et al. (2005) proposition that disks have been rebuilt following the encounters of gas-rich spirals. This proposition was inspired by the remarkable coincidence between the increase with redshift, up to $z=1$, in the fraction of actively star-forming galaxies (including LIRGs, i.e., luminous infrared galaxies) and the fraction of galaxies with anomalous morphologies, including the high 
fraction of luminous compact galaxies at $z \geq 0.4$, see Hammer et al. (2001, 2005). This proposition was followed by simulations of gas-rich mergers (Robertson et al. 2006; Governato et al. 2007), which demonstrated that a disk may be re-built after a merger. Such a conclusion had been already reached by Barnes (2002), who predicted: "these disks, if subsequently converted to stars, would be fairly hard to detect photometrically unless viewed from a favourable orientation." More recently Lotz et al. (2008b) analysed a large suite of simulated equal-mass, gasrich mergers and described most merger remnants as disc-like and dusty. In these simulations, significant disks may be rebuilt only if the encounters are very gas rich, with gas fraction of $50 \%$ or more. The importance of the evolution in the gas content of galaxies is still unknown because direct observations are out of reach before SKA. However, indirect observations based on the $\mathrm{O} / \mathrm{H}$ abundances of the gas phases of galaxies suggest high gas fractions at $z \sim 2$ (Erb et al. 2006), and even at $z \sim 0.6$ (Liang et al. 2006; Rodrigues et al. 2008).

Could we observe rebuilt disks and what would be their properties? If most of the mergers occur at $z=0.8-2$ (e.g., Ryan et al. 2008), a quite significant population of merger remnants should exist at $z=0.6$ or even at $z=0.4$ : the typical remnant timescale is found to be $\sim 2 \mathrm{Gyr}$, according to numerical simulations (e.g., Robertson et al. 2006), far longer than the characteristic time for a true pair to merge. As they should form stars rapidly, they are probably dusty and thus difficult to detect with moderately deep imagery. The optical disk of the Milky Way (defined to be 3.2 times the disk scale-length) would be detected out to $z=0.4-0.5$ (redshifted $R_{25}$ with $S / N \geq 3$ in a 1.5 resolution element) using HST/ACS imagery from GOODS. However, this does not apply for a disk enshrouded by dust, which can be detected only by far deeper exposures from the UDF. The VLT Large Program IMAGES (Yang et al. 2008) is observing a representative sample of $\sim 100 z=0.4-0.75$ intermediate-mass galaxies with $M_{J}(A B) \leq-20.3$. Four of these galaxies are located in the Hubble Ultra Deep Field, and for which we have the ultra-deep imagery from $B$ to $H$ band and kinematical data (from VLT/GIRAFFE), mid-IR data (from Spitzer) and deep spectroscopy (from VLT/FORS2). The HUDF galaxy analysed here is J033245.11-274724.0, at $z=0.43462$. In Sect. 2, we describe its overall properties, in Sect. 3 we present the data analysis and modelling, and in Sect. 4, we present our conclusions about the nature of this object. Throughout the paper, we adopt $H_{0}=70 \mathrm{~km} \mathrm{~s}^{-1} \mathrm{Mpc}^{-1}, \Omega_{\mathrm{M}}=0.3, \Omega_{\Lambda}=0.7$, and the $A B$ magnitude system.

\section{Properties of J033245.11-274724.0 at $z=\mathbf{0 . 4 3 4 6 2}$}

J033245.11-274724.0 is a very compact galaxy with $r_{\text {half }}=$ $1.7 \mathrm{kpc}$. It was firstly classified as a Tadpole due to its bright core and apparent coma-like shape (Neichel et al. 2008), on the basis of imaging data from GOODS. It is a luminous, massive galaxy with a stellar mass comparable or higher than that of the Milky Way. These compact objects have been already identified (Guzman et al. 1997; Hammer et al. 2001) to be quite numerous at intermediate redshift and far more abundant than in the present-day Universe (e.g. Rawat et al. 2007). Since they are often strong star-forming objects, their emergence at intermediate redshift contributes to the overall decline of the star formation density (e.g. Guzman et al. 1997).

Table 1 describes the overall properties of the galaxy, including photometric data, morphological parameters, kinematical data and long-slit spectral measurements. Photometric, kinematical and spectroscopic measurements are provided by the
Table 1. Properties of J033245.11-274724.0.

\begin{tabular}{|c|c|c|c|c|c|c|c|}
\hline \multicolumn{8}{|c|}{ Multi wavelength photometry, stellar mass and SFR } \\
\hline$M_{2800}$ & $M_{B}$ & $M_{V}$ & $M_{K}$ & $\begin{array}{l}M_{\text {stellar }} \\
\log \left(M_{\odot}\right)\end{array}$ & $\begin{array}{l}S F R_{2800} \\
M_{\odot} / \mathrm{yr}\end{array}$ & $\begin{array}{l}f_{24 \mu} \\
\mathrm{mJy}\end{array}$ & $\begin{array}{l}S F R_{\mathrm{IR}} \\
M_{\odot} / \mathrm{yr}\end{array}$ \\
\hline-18.71 & -20.12 & -20.76 & -22.05 & 10.8 & 1.9 & 0.353 & 21.7 \\
\hline \multicolumn{8}{|c|}{ Morphology: evaluated in observed $z$ band (equivalent to rest-frame $R$ band) } \\
\hline $\begin{array}{l}r_{\text {half }} \\
\mathrm{kpc}\end{array}$ & $B / T$ & $D / T$ & $\begin{array}{l}r_{\text {bulge }} \\
\mathrm{kpc}\end{array}$ & $\begin{array}{l}r_{\text {disk }} \\
\mathrm{kpc}\end{array}$ & $\begin{array}{l}\mu_{0, \text { disk }} \\
\text { mag/"2 }\end{array}$ & $\begin{array}{l}\text { incl }_{\text {disk }} \\
\text { deg }\end{array}$ & $\begin{array}{l}\mathrm{PA}_{\text {disk }} \\
\text { deg }\end{array}$ \\
\hline 1.7 & 0.14 & 0.51 & 0.13 & 1.45 & 20.4 & 39 & -66 \\
\hline \multicolumn{8}{|c|}{ Kinematics: from GIRAFFE measurements (Puech et al. 2008; Yang et al. 2008) } \\
\hline $\begin{array}{l}V_{\text {flat }} \\
\mathrm{km} \mathrm{s}^{-1} \\
290\end{array}$ & $\begin{array}{l}\sigma \text { (disk) } \\
\mathrm{km} \mathrm{s}^{-1} \\
74\end{array}$ & $\overline{v / \sigma}$ & $\begin{array}{l}j_{\text {disk }} \\
\mathrm{km} \mathrm{s}^{-1} \times \mathrm{kpc} \\
841\end{array}$ & $\begin{array}{l}\text { PA } \\
\text { deg } \\
70\end{array}$ & & & \\
\hline \multicolumn{8}{|c|}{ Spectroscopy } \\
\hline $\begin{array}{l}W_{0}(\mathrm{OII}) \\
\AA \\
15\end{array}$ & $\mathrm{H} \alpha / \mathrm{H} \beta$ & $c_{\mathrm{ext}}$ & $R_{23}$ & $\mathrm{O} / \mathrm{H}$ & $\begin{array}{l}\mathrm{OI} \\
/ \mathrm{H} \alpha \\
0 \mathrm{O4}\end{array}$ & $\begin{array}{l}\mathrm{NII} \\
/ \mathrm{H} \alpha \\
04\end{array}$ & $\begin{array}{l}S F R_{\mathrm{H} \alpha} \\
M_{\odot} / \mathrm{yr} \\
315\end{array}$ \\
\hline 15 & 6.2 & 1.0 & 1.9 & 9.1 & 0.04 & 0.4 & \\
\hline
\end{tabular}

IMAGES database and can be retrieved from Yang et al. (2008), Neichel et al. (2008), Puech et al. (2008) and Rodrigues et al. (2008). We note that the kinematics of compact galaxies such as J033245.11-274724.0 are affected by the large pixel size of GIRAFFE, and simulations of corresponding data-cubes reveal that large correction factors (1.5-2, see Puech et al. 2006; and Figure 5 of Puech et al. 2008) may be needed to recover the flat part of the rotation curve: this is due to the fact that the relatively coarse spatial resolution of GIRAFFE observations results in a smearing of the velocity gradient within each pixel.

J033245.11-274724.0 is an LIRG producing an enormous amount of stars, given its small size. We can estimate the global extinction of the system. Using the comparison of $S F R_{I R}$ with SFRs from $\mathrm{H} \beta$ and $\mathrm{H} \alpha$ provides $c_{\text {extinction }}=1.0$ for both Orion and standard extinction curves. This value appears to be robust, since it coincides with the value obtained from $\mathrm{H} \alpha / \mathrm{H} \beta$ and $\mathrm{H} \beta / \mathrm{H} \gamma$ ratios. Using the ratio of SFRs estimated at $2800 \AA$ to SFR in the infrared, we find $c_{\text {extinction }}=0.54$ and 0.79 , for the standard and the Orion extinction curves, respectively. This may favour the Orion extinction curve, and the contamination of the $2800 \AA$ continuum by an intermediate-age population may explain the differences between the extinction estimates. The overall galaxy color is $(b-z)_{A B}=2.28$, which at this redshift corresponds to that of a Sbc galaxy (see Fig. 8 of Neichel et al. 2008). Applying the Orion extinction curve to the overall color of the galaxy resolves most of the discrepancy due to the gap between Sbc and pure starburst colours $\left((b-z)_{A B}=0.78\right)$. A similar exercise assuming the standard extinction curve would provide a dereddened colour bluer than that of a pure starburst, which is not plausible. In the following, we adopt an Orion extinction curve with $c_{\text {extinction }}=1.0$.

Figure 1 compares the GOODS and UDF images and illustrates how vital the depth of UDF is for identifying the faintest structures in a dust-enshrouded galaxy. The global appearance of the galaxy consists of a central bulge with a small bar prolonged by very luminous arms, which are surrounded by a red disk. We used GALFIT to decompose the $z$-band galaxy luminosity profile (approximately rest-frame $R$ ) in individual components. The result is illustrated in Fig. 2. Given the appearance of the galaxy, we adopted a 3 component fitting (bulge, disk and arms) as provided by GALFIT. During our analysis, we attempted to vary some parameters such as the bulge Sersic index or its axis ratio. All of our attempts confirmed the numbers displayed in Table 1, namely the $\mathrm{B} / \mathrm{T}$ and $\mathrm{D} / \mathrm{T}$ ratio, disk and bulge radii, and disk inclination and PA. The smallest residual is obtained for a low value of the bulge Sersic index $(n=0.36)$, but it assumes a very inclined system $(b / a=0.07)$ a result which is likely affected by the bar. By assuming a round bulge we derived a Sersic index 


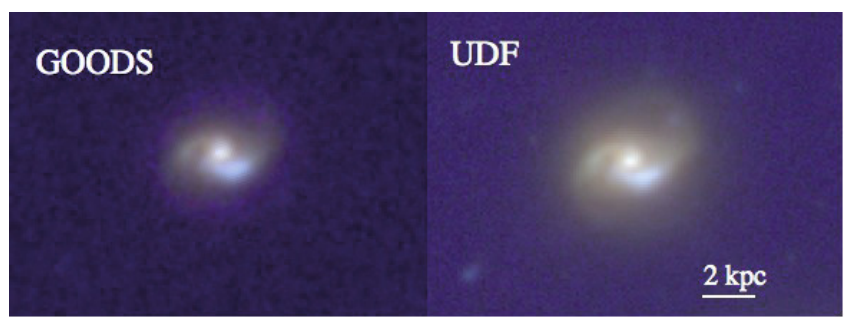

Fig. 1. $(b, v+i, z)$ colour-combined image of J033245.11-274724.0 from GOODS (left) and UDF (right), assuming the same $S / N$ background cut-off $(S / N>3)$. Only the depth of the UDF imagery allows us to derive robust parameters for the disk (PA, inclination, size): even at $z=0.4$, dusty disks require extremely deep exposures for such measurements. Notice the two small arms surrounding the bar near the centre that show a strong color difference suggesting the important role of dust extinction. The figure in the electronic version has a higher contrast.

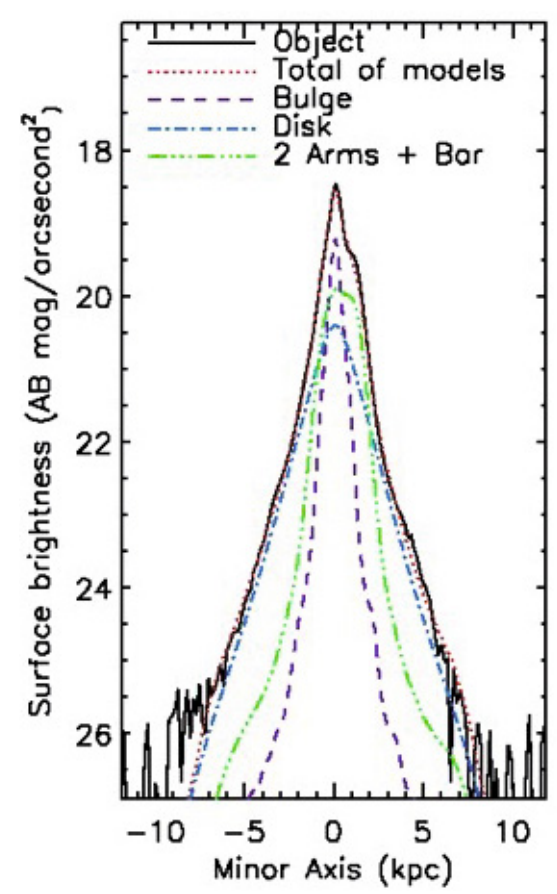

Fig. 2. Luminosity profile along the minor axis from the UDF $z$ band image showing the relative contributions of the bulge, disk and barredarms. This profile was obtained by assuming a round bulge with a Sersic index of 4 and a disk with a Sersic index of 1 .

of $n=3.89$, in good agreement with expectations for a classical bulge. We also attempted to vary the Sersic index of the disk, which provided a best fit solution for $n=0.97$ in good agreement with expectations for a exponential disk. For reason of simplicity, we then assumed values of $n=4$ and 1 for deriving the luminosity profile of the bulge and the disk, respectively (Fig. 2).

The UDF imagery (Fig. 1) reveals a red disk surrounding a blue bulge, which is quite unusual. The disk colour ranges from $(b-z)=2.8$ to 3.5 , i.e. close to that of very old stellar populations. Thus, it is likely that the disk of the LIRG is heavily extincted. Indeed, while the bar is clearly identifiable between the bluest arm and the bulge, it vanishes on the other side. The dust within the disk may also be responsible for the colour asymmetry of both bar and arms, such that the bluest arm is in the foreground to the disk, while the other is in background. It suggests another oddity of J033245.11-274724.0: the arms - as well as the bar - may not be located fully inside the disk, so that a
Table 2. Decomposition into multiple components.

\begin{tabular}{|c|c|c|c|c|c|c|c|}
\hline \multicolumn{8}{|c|}{ Observed magnitudes - extinction (assuming late type disk color) - corrected values } \\
\hline Component & $z$ & $b-z$ & $\begin{array}{l}c_{\text {ext }} \\
\text { /starburst }\end{array}$ & $\begin{array}{l}c_{\text {ext }} \\
\text { assum. }\end{array}$ & $b_{\mathrm{c}}$ & $S F R_{\mathrm{c}}$ & $M_{\text {stellar }}(\mathrm{c})$ \\
\hline & mag & mag & Or. & & mag & $M_{\odot} / \mathrm{yr}$ & $\log \left(M_{\odot}\right)$ \\
\hline bulge & 22.5 & 2.0 & 1.13 & 0 & 24.5 & 0.3 & 8.9 \\
\hline disk & 21.13 & 2.9 & 2.15 & 1.28 & 19.94 & 20.1 & 10.52 \\
\hline foreg. arm & 22.0 & 1.6 & 0.76 & 0 & 23.6 & 0.65 & 9.1 \\
\hline backg. arm & 22.74 & 2.3 & 1.41 & 0.65 & 23.6 & 0.65 & 9.1 \\
\hline total & 20.42 & 2.28 & 1.4 & 1.0 & 19.42 & 21.7 & 10.61 \\
\hline
\end{tabular}

significant part of them emerges above and below the disk. Both arms have clear extensions that surround the disk, suggesting spiraling motions from the disk edge to the centre (see Fig. 1). Gas motions are quite complex, since the main disk photometric axis is severely offset from the dynamical axis (see Table 1).

We note that J033245.11-274724.0 has been classified by (Yang et al. 2008) as a rotating basis on the sole basis of its kinematics, in absence of a clear detection of the dust-enshrouded disk (see Fig. 1). The detection of the disk with UDF imaging challenges this classification because of the misalignment between optical and dynamical axes. This also explains why several quantities in Table 1 differ from those estimated earlier: in this study, the $B / T$ ratio and the disk inclination (and thus derivation of the velocity) were calculated after applying GALFIT to UDF rather than to GOODS imagery.

\section{Physical model of J033245.11-274724.0}

\subsection{De-reddened properties reveal a dominant dust-enshrouded disk}

The bulge has a colour intermediate between a Sbc and a latetype galaxy, and the bluest arm has a colour similar to that of a late type galaxy. The oldest stars in the system are likely to inhabit the bulge, and it is reasonable to assume that both the bulge and the bluest arm are almost not affected by dust. By using NICMOS observations in the $J$ and $H$ band (rest-frame near IR), we find that the $B / T$ ratio does not vary significantly from our fiducial value ( 0.14 ; see Table 1$)$, meaning that there is no significant dust-enshrouded component in the bulge. Table 2 presents the decomposition of the galaxy into multiple components assuming that the extinction affects mostly the red disk component as well as the background arm. In this model, the disk dominates J033245.11-274724.0, representing 81\% of its stellar mass and more than $90 \%$ of its star formation. The appearance of the dereddened galaxy would be a disk/arm system with the colour of a late-type galaxy $(b-z=1.5-1.6)$ surrounding a bulge with a colour $(b-z=2)$ intermediate between a Sbc and a late-type spiral. Assuming a uniform colour for all components would not affect our conclusion that J033245.11274724.0 is heavily dominated by its disk, both in terms of mass and star formation. We note that the corrected stellar mass of J033245.11-274724.0 was estimated from the rest-frame $K$-band magnitude (after correction for extinction) and was assumed the extinction-corrected $B-V$ colour in estimating the $M_{\text {stellar }} / L_{K}$ ratio (see Hammer et al. 2005).

\subsection{Spectral properties: young and intermediate age stars dominate}

We also obtained deep spectroscopy with FORS2 (Rodrigues et al. 2008). After correction for extinction (Fig. 3), the spectrum is dominated by the light of young and intermediate age 


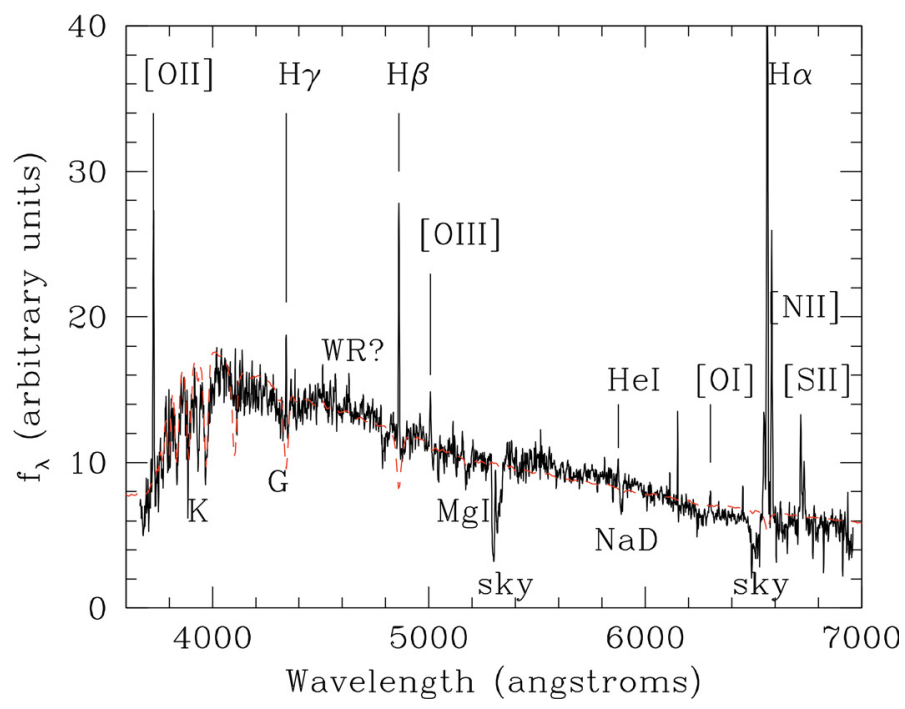

Fig. 3. FORS2 spectrum of J033245.11-274724.0 after dereddening the continuum by $c_{\text {extinction }}=1.0$ (Orion extinction curve). The spectral energy distribution can be reproduced (red line) by a set of models with stellar ages t from 0.25 to $1.2 \mathrm{Gyr}$ assuming $\tau(S F R \sim \exp (-t / \tau))$ from 0 to 5 Gyr.

(A/F) stars, as indicated by the strong emission lines and strong Balmer absorption lines $(E Q W(\mathrm{H} \delta)=4.5 \AA)$. The line ratios are consistent with those of HII galaxies. There is some evidence that extremely young and hot stars are present as indicated by the detection of the He $5875 \AA$ line, and possibly WolfRayet features, such as the blue bump and possible detection of [SiIII]4565] $\mathrm{A},[\mathrm{NV}] 4620 \AA$, [HeII]4686 $\mathrm{A}$, and [NII]5720 . The global metal abundance is over-solar, at $12+\log (\mathrm{O} / \mathrm{H})=$ 9.1 , which can be explained by the very large density of recent and past star formation all over the galaxy. The presence of metal absorption lines (CaIIK, G, MgI \& NaD) indicates the presence of relatively old stars. We modeled the stellar populations using the STARLIGHT code (e.g. Cid-Fernandes et al. 2008). The high quality of the FORS2 spectrum is at least comparable to that of a typical galaxy from the SDSS. It provides a distribution of stellar age including a large mass-component of young to intermediate age stars (mass scaled: $20 \%$ with age $\leq 0.3 \mathrm{Gyr}$, $33 \%$ with age $=0.5-0.8 \mathrm{Gyr}$ ), the remainder being associated with older ages ( $>2 \mathrm{Gyr}$ ). This confirms the recent formation of a significant part of the stellar mass in J033245.11-274724.0.

\subsection{Morpho-kinematic properties: a hot disk out of equilibrium}

J033245.11-274724.0 is dominated by a dust-enshrouded disk with a quite peculiar two-armed system. The kinematical axis is strongly offset from the photometric major axis of the disk and is slightly offset from the galaxy centre (Fig. 4, upper panels), arguing for a non-axisymmetric, heavily perturbed and out of equilibrium disk. The outskirts of J033245.11-274724.0 exhibit extremely high velocity dispersions, ranging from 60 to $105 \mathrm{~km} \mathrm{~s}^{-1}$ (Fig. 4 and Yang et al. 2008). Puech et al. (2007) estimated the intrinsic velocity dispersion of the disk by excluding the central pixels, which are likely to be affected by the central velocity gradient linked with the rotation at low spatial resolution. By excluding the central regions, Puech et al. (2007) found $\sigma_{\text {disk }}=74 \mathrm{~km} \mathrm{~s}^{-1}$ for J033245.11-274724.0, a value far larger than expected for a thin disk. The disk clearly dominates the
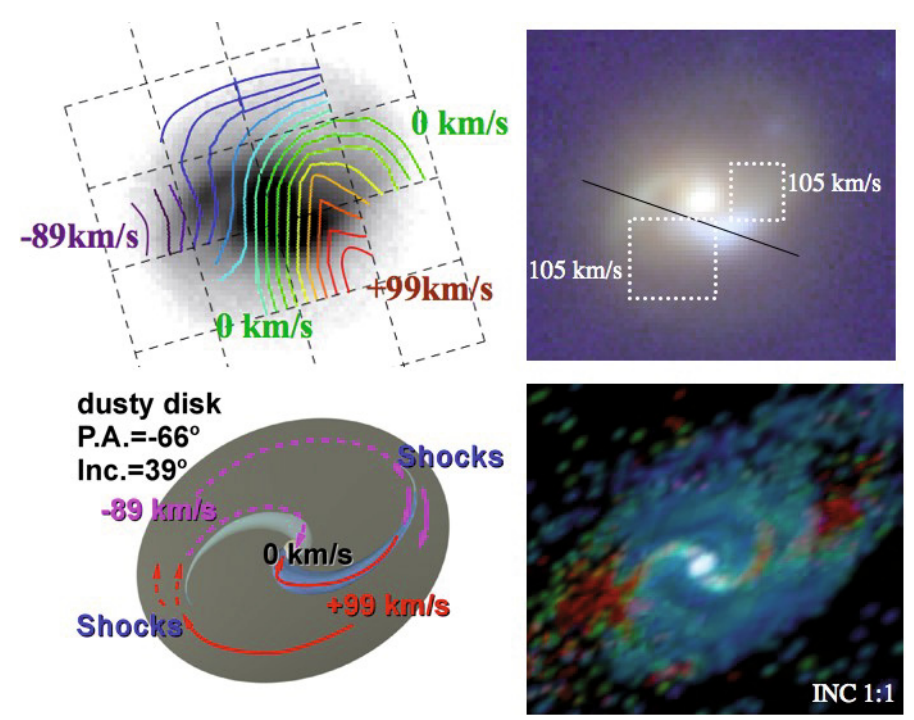

Fig. 4. Upper left: $v$-band image of J033245.11-274724.0 on which the GIRAFFE iso-velocities have been superimposed. Colour coding is the same as in Yang et al. (2008). Upper right: $b-v+i-z$ colourcombined image of J033245.11-274724.0 on which the dynamical axis (full lack line) and the velocity dispersion peaks (dotted squared boxes) at $105 \mathrm{~km} \mathrm{~s}^{-1}$ have been superimposed. The entire galaxy shows large velocity dispersion (Yang et al. 2008). The figure in the electronic version is of higher contrast. Bottom left: a sketchy model of the galaxy showing the dusty disk, and the gas motions (purple: approaching; red: recessing) from the background (dotted lines) to the foreground of the disk (full lines). The bar close to the centre is inclined with respect to the disk plane, explaining its disappearance at the top-right of the bulge as well as the asymmetry between the two arms. The resulting dynamical axis is thus caused by the gas motion both through the bar and around the disk. Bottom right: result of a galaxy collision (1:1 mass ratio) with an inclined orbit (close encounters) from Barnes (2002), 0.5 Gyr after the merger. The gas wraps up inside the disk, creating a bar and a symmetric-arm system whose motion does not follow the gas rotation, creating shocks and high velocity dispersions (seen as red particles in the simulation), which are consistent with observations.

outskirts of the galaxy as it can be inferred from the luminosity profile (Fig. 2), and thus, the bulge cannot bring any significant contribution to the high dispersion.

We also note that the velocity dispersion peaks are located near the ends of the arms, which is rather unexpected for the velocity field of a rotating disk observed at low spatial resolution (Flores et al. 2006). Figure 4 (bottom-left) shows a sketchy representation of the galaxy, which reproduces both its morphology and kinematics.

The disk of J033245.11-274724.0 is rotating too fast for its stellar mass. Indeed, assuming a disk inclination of $39^{\circ}$ (see Table 1), the fitting of the velocity field (Puech et al. 2008) provides $V_{\text {flat }}=290 \mathrm{~km} \mathrm{~s}^{-1}$, while at its stellar mass, the TullyFisher relation predicts $V_{\text {flat }}=190 \mathrm{~km} \mathrm{~s}^{-1}$ at $z=0$. This shift relative to the Tully-Fisher relation is significant, and cannot be due to an underestimate of the inclination: adopting $V_{\text {flat }}=$ $190 \mathrm{~km} \mathrm{~s}^{-1}$ would infer a disk inclination of $74^{\circ}$, which is clearly inconsistent with observations. It is thus likely that the disk which actively forms stars at a rapid rate - would increase its stellar mass further, and would evolve into agreement with the local Tully-Fisher at $z=0$. 

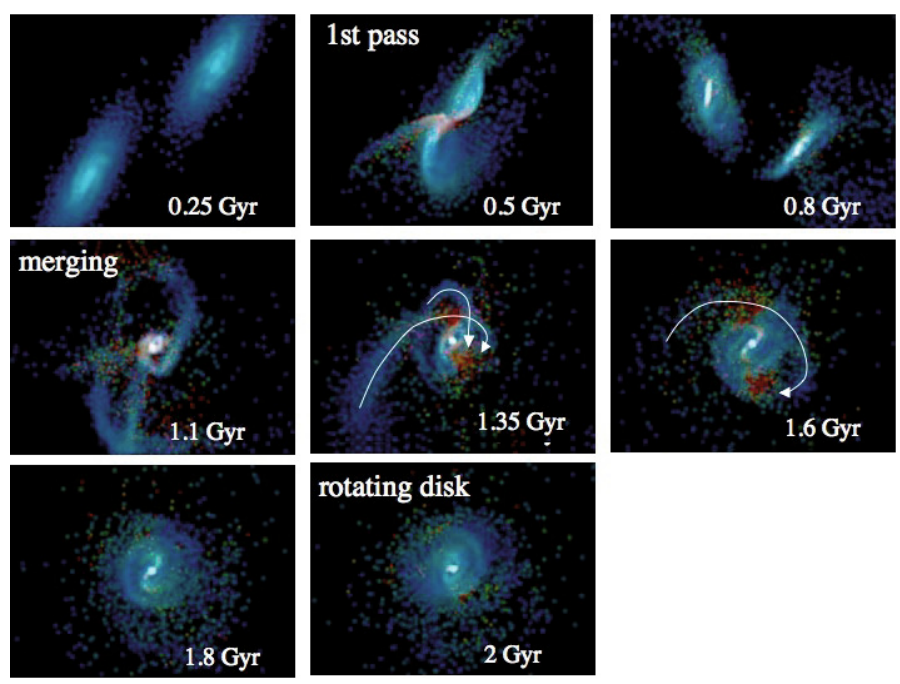

Fig. 5. Inclined-orbit model from Barnes (2002) with time scaled for a fusion of two galaxies with half the mass of the Milky Way. The arrows in the central panels (at 1.35 and $1.6 \mathrm{Gyr}$ ) indicate the gas motions and the exchanges of angular momentum after the merger. The orbital angular momentum is perpendicular to the observed plane, in contrast to the angular momentum of the progenitors (see panels at 0.25 and $0.8 \mathrm{Gyr}$ ), resulting in a face-on rotating disk at the end of the simulation. The bar and two armed system regulate the final disk rotation. Note that the two armed system does not rotate (compare panels at 1.6 and $1.8 \mathrm{Gyr}$ ).

\subsection{Comparison to simulations: a merger remnant?}

Similar features (bar + two armed system) are observed in current simulations of galaxy mergers well after the collision (see Fig. 4, bottom-right). These features do not follow the circular gas motions ${ }^{1}$, and their misalignment with respect to the disk may be due to the fact that they originate preferentially in one of the two progenitors (see Barnes 2002). Their main role is to funnel gas towards the galaxy to fuel the formation of the bulge and the disk. After disk stabilisation, they may disappear in the simulation. The global resemblance between the simulations and the observations (morphology and kinematics) is rather striking. $N$-body/SPH simulations by Barnes (2002) use a minimum of prescriptions that may simplify this comparison by minimising the number of physical ingredients. The predicted rebuilt disks in Barnes (2002) are indeed warped, and the kinematics of J033245.11-274724.0 (kinematical axis strongly offset from the photometric axis \& velocity dispersion peaks) is clearly reproduced by simulations. We note that the example shown in Fig. 4 illustrates the gas component only. It can, nevertheless, be compared to the observed stellar distribution, since most of the emission in the J033245.11-274724.0 system should be dominated by young and massive stars, of ages relatively small in comparison with dynamical timescales.

\section{Discussion}

The wealth of data for J033245.11-274724.0 has allowed us to identify a faint, red disk that dominates both its star formation and stellar mass. The disk is indeed small with a radius half that of the Milky Way, while having a similar stellar mass and forming stars 20 times more rapidly. The presence of a bar close to the

1 We encourage the reader to examine the simulation at http://www . ifa.hawaii.edu/\$ sim\$barnes/research/gassy_mergers/ index.html, model with 1:1 mass ratio, INClined orbit and pericenter $r_{\mathrm{p}}=0.2$. centre, a symmetrical two-arm system extending on both sides of the disk plane towards the disk edge, is indicative of a significant spiraling motion of both gas and stars from the outskirts to the centre. Our sketchy representation of J033245.11-274724.0 reproduces well the morphological features as well as the kinematics, assuming that the symmetric arms do not follow the circular gas motions, and thus create shocks that are revealed by the observed velocity dispersion peaks in the $2 \mathrm{D}$ velocity dispersion map. The overall properties of J033245.11-274724.0 are thus consistent with the modeling of the remnant of a merger of two gaseous, equal-mass disks on an inclined orbit. It appears that by combining deep UDF imaging with GIRAFFE integral field spectroscopy, we may have revealed the presence of a dustenshrouded disk that is also a merger remnant.

\subsection{A perturbed disk or a merger remnant?}

Does the success of our physical model in reproducing the observed features necessarily imply that J033245.11-274724.0 is a merger remnant? Before reaching this conclusion, we examine some possible alternatives. Firstly, the evidence that the system is disturbed and not in equilibrium might be explained by a passing, tidal interaction. However, investigations of the field surrounding J033245.11-274724.0 do not reveal any possible companions within several hundreds of kpc, using numerous existing spectroscopic or photometric redshifts in the field available for all galaxies with apparent luminosities down to one third of that of J033245.11-274724.0. Examining the UDF imagery in $z$ band, the brightest nearby galaxy (with no redshift available) within $50 \mathrm{kpc}$ has a $z$-band luminosity that is $1 / 15$ th that of J033245.11274724.0. Within $30 \mathrm{kpc}$, the brightest nearby companion has a z-band luminosity that is $1 / 200$ th that of J033245.11-274724.0. It is thus very unlikely that a fly-by interaction may be the cause of the exceptional properties of this galaxy.

Secondly, internal processes related to the starburst may be considered to explain its properties. In particular, the fact that the kinematical axis is severely offset from the photometric axis may be indicative of gas super-winds (outflows) induced by supernova. These phenomena are observed in many starbursting galaxies (e.g. Heckman \& Lehnert 2000). We tested such an alternative by comparing the velocity of the emission-line system with that of the absorption-line system, and by examining the line profile of the $\mathrm{NaD}$ absorption lines, i.e. the two methods outlined by Heckman \& Lehnert (2000). By measuring ten robust emission lines $(\mathrm{H} \gamma, \mathrm{H} \beta$, [OIII]4959 and 5007, HeI, [OI], [NII]6548 and 6583 and $\mathrm{H} \alpha$ ), we find a remarkable agreement between those lines with a scatter of only $15 \mathrm{~km} \mathrm{~s}^{-1}$. We excluded the two [SII] lines, since they exhibit a much larger shift, presumably due to the fact that they lie at the edge of the spectrum, where wavelength calibration may have failed. Examining the absorption lines of a reasonable $S / N$ (Balmer H12 and H11, CaII K, CaI, NaI-D1 and D2), we find a larger scatter of $52 \mathrm{~km} \mathrm{~s}^{-1}$ as expected. The absorption and emission line system appear to have the same velocity, to within an uncertainty of $33 \mathrm{~km} \mathrm{~s}^{-1}$, a value far below the expected uncertainties dominated by absorption-line measurements (recall that the FWHM spectral resolution is $450 \mathrm{~km} \mathrm{~s}^{-1}$ ). The contribution of the ISM to the NaD lines may be evaluated following Heckman \& Lehnert (2000) from the expected relationship for typical stellar populations: $\mathrm{EQW}(\mathrm{NaD})=0.75 \times \mathrm{EQW}(\mathrm{MgIb})$. For J033245.11-274724.0, we find EQW(NaD) $=2.8 \AA$ and $\mathrm{EQW}(\mathrm{MgIb})=4.0 \AA$, which implies a negligible contribution from the ISM. From both tests, J033245.11-274724.0 appears to 
Table 3. A comparative test of the perturbed disk and the merger remnant hypotheses.

\begin{tabular}{|c|c|c|}
\hline Observed property & Best hypothesis & Comments \\
\hline much redder disk than the bulge & merger remnant & rapid disk rebuilt is predicted (Governato et al. 2007): renewed disks are thus star forming and dusty (Lotz et al. 2008b) \\
\hline misaligned dynamical axis & merger remnant & in absence of significant outflow, it reveals a system not at equilibrium, difficult to explain except by a merger \\
\hline average disk velocity dispersion $\left(74 \mathrm{~km} \mathrm{~s}^{-1}\right)$ & merger remnant & unclear which perturbation other than merging can provide such a hot disk $\left(\sigma>56 \mathrm{~km} \mathrm{~s}^{-1}\right)$ \\
\hline velocity dispersion peak locations & merger remnant & only two minor mergers near the two locations may explain such features in a perturbed disk \\
\hline very high disk star formation density & merger remnant & regulation of star formation between gravitational instabilities and porosity is expected in rotating disks (e.g. Silk 1997) \\
\hline discrepancy from the Tully Fisher & merger remnant & pected in a merger (e.g. Puech et al. 2007) and quite unexpected in a perturbed disk (see above) \\
\hline half of the stars with ages lower than $800 \mathrm{Myr}$ & merger rer & correspond to the merger time-scale during which these stars are formed \\
\hline
\end{tabular}

be part of the Heckman \& Lehnert (2000) sample of "strong stellar contamination" starbursts, i.e. those showing no evidence of outflows. Examination of the emission-line profiles also show no evidence of an outflow. An absence of a significant outflow contribution is unsurprising given the high mass of the galaxy, whose gravitational forces prevent stellar super-winds dominating the velocity field.

Given the above, we have no suitable explanations of J033245.11-274724.0 other than the merger-remnant hypothesis. Table 3 illustrates this by presenting the two alternatives, a perturbed disk and a merger remnant, and testing them against the main observed properties.

\subsection{A merger remnant of a $1: 1$ or a $1: 3$ fusion?}

Does the success of our physical model necessarily imply that J033245.11-274724.0 is a merger remnant of a 1:1 mass ratio merger?

Using simulations by Barnes (2002), we also tested a model with 3:1 mass ratio. While this also predicts a shape similar of the "bar plus 2 arms system", it is unable to reproduce both the morphology and the high velocity dispersion peaks of $105 \mathrm{~km} \mathrm{~s}^{-1}$. On the other hand, we tried to reproduce the Barnes (2002) simulations of $1: 1$ and 1:3 mass ratio using the same parameters but with the Gadget 2 code (Springel 2005). A bar is still present (and persistent), but with a less pronounced $\mathrm{S}$ shape. Different recipes of gas viscosity and star formation may explain these discrepancies. Admittedly, it is beyond the scope of this paper to provide an accurate prediction of the mass ratio of the progenitors of J033245.11-274724.0, although existing simulations clearly favour a range from $1: 1$ to $1: 3$.

It is also important to note that the simulations by Barnes (2002) predict that a significant fraction of the gas lies in the remnant nuclei and not in the disk. On the other hand, in the Lotz et al. (2008b) realisations several merger remnants have the appearance of Sbc galaxies. A full quantitative model of this source would be highly desirable to investigate the formation stage of the J033245.11-274724.0 disk. For example, Hopkins et al. (2008) generated a full suite of galaxy-galaxy simulations to examine the properties of their corresponding remnants. Their simulations may include J033245.11-274724.0 as suggested by their abstract: "the efficiency of disk destruction in mergers is a strong function of gas content - our model allows us to predict explicitly and demonstrate how, in sufficiently gas-rich mergers (with quite general orbital parameters), even 1:1 mass-ratio mergers can yield disk-dominated remnants, and more realistic 1:3-1:4 mass-ratio major mergers can yield systems with $<20 \%$ of their mass in bulges." Motivated by this, we attempted to estimate the gaseous content of the galaxy by assuming that it follows the Schmidt-Kennicutt relation between star formation and gas surface densities (e.g. Kennicutt 1998). For this, we further assumed that the star-formation density could be derived from a surface radius of $2 \times r_{\text {half }}=3.4 \mathrm{kpc}$, and that the gas surface density could be derived from the maximal extent of the $[\mathrm{OII}]$ emission detected by GIRAFFE $\left(r_{\mathrm{OII}}=5.4 \mathrm{kpc}\right.$ after deconvolution from the natural seeing, $F W H M=0.8$ arcsecond). We then measured a star formation surface density of $0.6 M_{\odot} \mathrm{yr}^{-1} \mathrm{kpc}^{-2}$, a gas mass density of $2.5 \times 10^{8} M_{\odot} \mathrm{kpc}^{-2}$ and a high total gas mass of $2.310^{10} M_{\odot}$. Assuming the stellar mass derived in Table 2 (after accounting for dust extinction effects), we reached the conclusions that the J033245.11-274724.0 system may include up to $37 \%$ of gas. Since a significant fraction of the stars (approximately half, see Sect. 3.2) may have been formed during the interaction, the gas fraction in the progenitors of J033245.11-274724.0 may had been much higher, up to $67 \%$. Such a value would be consistent with significant disk rebuilding as demonstrated by the Robertson et al. (2006) simulations.

We thus are left with an Occam's razor-type argument: the overall and detailed properties of J033245.11-274724.0 are well reproduced by a major merger remnant (Table 3), we do expect the existence of such a remnant from interactions between gasrich galaxies, and such an event may be quite common given existing merger statistics, while other explanations require the combination of unknown mechanisms. We thus conclude that J033245.11-274724.0 may be the first conclusive example of a rebuilt disk in the intermediate redshift Universe.

\section{Concluding remarks}

J033245.11-274724.0 is a warped disk that is dust enshrouded and forms stars at a very high rate, given its small size. Examination of the exchanges of angular momentum in Barnes (2002) simulation is instructive (Fig. 4). Before the second impact, the angular momentum of the two equal mass progenitors are almost perpendicular to that of the newly formed disk, whose plane is determined by the orbital angular momentum. After the second impact, the gas and stars roll up surrounding the newly formed bulge following trajectories that result from their initial angular momentum combined with the tidal forces. The twoarmed system plays the role of the bar, redistributing the angular momentum of the infalling material towards the bar and then to the bulge (by means of dispersion) or towards the newly formed disk. At the stage represented by J033245.11-274724.0, most of the stars and most of the newly formed stars are located in the forming disk. This transition is quite long, since the bar plus non rotating two-armed system are seen for about $20 \%$ of the total time in the simulation (Barnes 2002). The total time of the simulation is approximately 2 Gyr for encounters with half the Milky Way mass, the first impact occurring at $0.5 \mathrm{Gyr}$, the merging of the nuclei occurring at $1.1 \mathrm{Gyr}$, the appearance of the non rotating two-armed system at $1.6 \mathrm{Gyr}$ and its disappearance at $1.9 \mathrm{Gyr}$. These numbers are quite consistent with the ages of the stellar populations that dominate the J033245.11-274724.0 continuum. On the other hand, the creation of a disk with $3.7 \times$ $10^{10} M_{\odot}$ would require $1.8 \mathrm{Gyr}$ at the rate of $20 M_{\odot} / \mathrm{yr}$ (see Table 2), i.e. a time that is long compared to the time it takes the 
disk to settle after the merger in the simulation $(<0.5 \mathrm{Gyr})$. The observed stellar mass in the J033245.11-274724.0 disk of course consists of young stars, although a significant part consists of intermediate-age stars, probably produced during the first stages of the merger, and possibly of older stars accreted from the progenitors. The colour of the dereddened disk (late type galaxy) is consistent with such a mix of stellar population.

What will the final product of J033245.11-274724.0 be? Since most of the star formation has been induced in the disk, one could assume a progressive inside-out disk-building from the residual material accreting onto the disk. As the infalling gas becomes rarer, the star formation and the dust production will decrease and the galaxy will take the appearance of a blue star forming disk. In its further evolution to reach the local TullyFisher relation, J033245.11-274724.0 needs to form a larger and more massive disk, which may be followed by bulge growth by gas accretion from the residual bar. Assuming that the $37 \%$ residual gas fraction estimated in Sect. 4.2 will have been transformed into stars by $z=0$, the galaxy may reach the local Tully Fisher relationship. The observed bar in J033245.11-274724.0 is very small (and blue, see Fig. 1) and the simulation does predict a residual small bar a few tenths of Gyr later. Our observations show that most of the stellar mass is in the disk: it is probable that the newly formed system would have a bulge to total ratio ranging from 0.03 to 0.09 (e.g. Sc or later type), depending on whether the material in the two-armed system will be deposited in the disk or in the bulge, respectively. Thus, our observations and modeling of J033245.11-274724.0 are highly indicative of the formation of a massive, late-type spiral whose angular momentum support is mostly provided by the orbital momentum of the last merger.

It is quite a challenge to find similar examples in the local Universe, which is moreover lacking LIRGs $(0.5 \%)$, compact luminous galaxies (a few percents, Rawat et al. (2007)) and, overall, blue bulges at the centre of extremely red disks. NGC 1614 is a powerful LIRG and a merger remnant and shows some similarities with J033245.11-274724.0. However, in NGC 1614 most of the starburst activity is in the core (Alonso-Herrero et al. 2001), which is probably induced by the recent coalescence of the two progenitor nuclei (Neff et al. 1990, and see Fig. 4, panel at $1.1 \mathrm{Gyr}$ ): it seems to be in a less advanced merger stage than J033245.11-274724.0.

Finally, the occurrence of these merger remnants in the past may be far more common than today. Observed at relatively shallow depth (compared to UDF), J033245.11-274724.0 appears to be a Tadpole due to its bright blue arm. Tadpoles represents $2-5 \%$ of the massive galaxies at $z=0.6-1$ (Brinchmann et al. 1998; Neichel et al. 2008), and some might be similar to J033245.11-274724.0, possibly seen at different viewing angles. In the near future, we may be able to evaluate whether J033245.11-274724.0 is an isolated case or a more general phenomenon of disk rebuilding.

Acknowledgements. We are especially indebted to Josh Barnes for his comments and criticisms in reading an early version of the manuscript as well as for making publicly his simulations of galaxy mergers on his web pages. We are very grateful to the referee whose suggestions have helped a lot to improve the manuscript.

\section{References}

Alonso-Herrero, A., Engelbracht, C. W., Rieke, M. J., \& Quillen, A. C. 2001, ApJ, 546, 952

Barnes, J. E. 2002, MNRAS, 333, 481

Bell, E. F., Phleps, S., Somerville, R. S., et al. 2006, ApJ, 652, 270

Brinchmann, J., Abraham, R., Schade, D., et al. 1998, ApJ, 499, 112

Brown, T., Beaton, M., R., C., M., et al. 2008, ApJ, 685, 121

Eggen, O. J., Lynden-Bell, D., \& Sandage, A. R. 1962, ApJ, 136, 748

Erb, D. K., Shapley, A. E., Pettini, M., et al. 2006, ApJ, 644, 813

Fall, S. M., \& Efstathiou, G. 1980, MNRAS, 193, 189

Flores, H., Hammer, F., Puech, M., Amram, P., \& Balkowski, C. 2006, A\&A, 455,107

Governato, F., Willman, B., Mayer, L., et al. 2007, MNRAS, 374, 1479

Hammer, F., Gruel, N., Thuan, T. X., et al. 2001, ApJ, 550, 570

Hammer, F., Flores, H., \& Elbaz, D. 2005, A\&A, 430, 115

Hammer, F., Puech, M., Chemin, L., Flores, H., \& Lehnert, M. 2007, ApJ, 662, 322

Heckman, T., \& Lehnert, M. 2000, ApJ, 129, 493

Hopkins, P., Hernquist, L., Cox, T. J., Younger, J. D., \& Besla, G. 2008, ApJ, submitted [arxiv/0806.2861]

Ibata, R., Chapman, S., Ferguson, A. M. N., et al. 2005, ApJ, 634, 287

Liang, Y. C., Hammer, F., \& Flores, H. 2006, A\&A, 447, 113

Lotz, J. M., Davis, M., Faber, S.M., et al. 2008a, ApJ, 672, 177

Lotz, J. M., Jonsson, P., Cox, T. J., \& Primack, J., R. 2008b, MNRAS, submitted [arxiv/0805.1246]

Mo, H. J., Mao, S., \& White, S. D. M. 1998, MNRAS, 295, 31

Neff, S. G., Hutchings, J. B., Stanford, S. A., \& Unger, S. W. 1990, ApJ, 99, 1088

Neichel, B., Hammer, F., Puech, M., et al. 2008, A\&A, 484, 159

Puech, M., Hammer, F., Flores, H., Östlin, G., \& Marquart, T. 2006, A\&A, 455, 119

Puech, M., Hammer, F., Lehnert, M., \& Flores, H. 2007, A\&A, 484, 173

Puech, M., Flores, H., Hammer, F., et al. 2008, A\&A, 484, 173

Rawat, A., Kembhavi, A., Hammer, F., Flores, H., \& Barway, S. 2007, A\&A, 469,483

Rawat, A., Hammer, F., Kembhavi, A., \& Flores, H. 2008, ApJ, 681, 1089

Robertson, B., Bullock, J. S., Cox, T. J., et al. 2006, ApJ, 645, 986

Rodrigues, M., Hammer, F., Flores, H., et al. 2008, A\&A, 492, 371

Ryan, R., E., Cohen, S. H., Windhorst, R. A., \& Silk, J. 2008, ApJ, 678, 751

Silk, J. 1997, ApJ, 481, 703

Springel, V. 2005, MNRAS, 364, 1105

Steinmetz, M., \& Navarro, J. 1999, ApJ, 513, 555

Toth, G., \& Ostriker, J. P. 1992, ApJ, 389, 5

Yang, Y., Flores, H., Hammer, F., et al. 2007, A\&A, 477, 789 